\title{
An Efficiency Assessment for Reflectance Normalization of RapidEye Employing BRD Components of Wide-Swath Satellite
}

\author{
Sang-il Kim, Kyung-Soo Han* ${ }^{\dagger}$, Jong-Min Yeom** \\ *Dept. of Spatial Information Engineering, Pukyung National University, Daeyeon-3 Nam-Gu, Busan 608-737, Korea \\ **Satellite Information Research Institute (SIRI), Korea Aerospace Research Institute (KARI)
}

\begin{abstract}
Surface albedo is an important parameter of the surface energy budget, and its accurate quantification is of major interest to the global climate modeling community. Therefore, in this paper, we consider the direct solution of kemel based bidirectional reflectance distribution function (BRDF) models for retrieval of nomalized reflectance of high resolution satellite. The BRD effects can be seen in satellite data having a wide swath such as SPOT/NGT (VEGETATION) have sufficient angular sampling, but high resolution satellites are impossible to obtain sufficient angular sampling over a pixel during short period because of their narrow swath scanning when applying semi-empirical model. This gives a difficulty to run BRDF model inferring the reflectance normalization of high resolution satellites. The principal purpose of the study is to estimate normalized reflectance of high resolution satellite (RapidEye) through BRDF components from SPOT/VGT. We use semi-empirical BRDF model to estimated BRDF components from SPOT NGT and reflectance normalization of RapidEye. This study used SPOT/NGT satellite data acquired in the S1 (daily) data, and within this study is the multispectral sensor RapidEye. Isotropic value such as the normalized reflectance was closely related to the $\mathrm{BRDF}$ parameters and the kernels. Also, we show scatter plot of the SPOT VGT and RapidEye isotropic value relationship. The linear relationship between the two linear regression analysis is performed by using the parameters of SPOT/VGT like as isotropic value, geometric value and volumetric scattering value, and the kemel values of RapidEye like as geometric and volumetric scattering kernel. Because BRDF parameters are difficult to directly calculate from high resolution satellites, we use to BRDF parameter of SPOT/NGT. Also, we make a decision of weighting for geometric value, volumetric scattering value and error through regression models. As a result, the weighting through linear regression analysis produced good agreement. For all sites, the SPOTNGT isotropic and RapidEye isotropic values had the high correlation (RMSE, bias), and generally are very consistent.
\end{abstract}

Key Words: SPOT NGT, RapidEye, BRDF, isotropic value, geometric value, volumetric scattering value

Received May 27, 2011; Revised June 21, 2011; Accepted June 22, 2011.

${ }^{\dagger}$ Corresponding Author: Kyung-Soo Han (kyung-soo.han@pknu.ac.kr) 


\section{Introduction}

As the one of the major energy sources, incoming solar radiation plays a fundamental role in determining weather or climate at the atmospheric boundary layer by strong feedback effects (Pokrovsky and Roujean, 2002). This global climate change is undergoing and it is important to exactly understand form and status of surface for monitoring climate change. Consequently, knowledge of the phenology of plant communities is relevant to estimate biological productivity, understanding landatmosphere interactions and biome dynamics, modeling vegetative inputs into biogeochemical cycles, as well as for the management of vegetation resources (Tarpley et al., 1984; Justice et al., 1985). Also, land surface albedo is defined as the fraction of incident solar irradiance reflected by Earth's surface over the whole solar spectrum (Dickinson, 1983). Especially, wide-swath satellite such as AVHRR, MODIS, and SPOT/VGT (VEGETATION) has anisotropy effects that can cause serious error in estimations of solar radiation parameters such as reflectance, albedo and vegetation indices (Yeom et al., 2005; Yeom et al., 2009). The anisotropic behavior of surface reflectance is described by the Bidirectional Reflectance Distribution Function (BRDF). The BRDF can be used to model observed reflectance to the normalized reflectance at nadir (Kimes et al., 1985; Roujean et al., 1992). Therefore, the BRDF is considered as one of the most important factors when utilizing satellite data and deriving biophysical information from the ground surface (Chopping, 2000; Wanner and Strahler, 1995). Also, this model has been optimized to base on the wideswath satellite which can acquire sufficient angular sampling for a short time. There are several kinds of BRDF models, mainly utilizing physical (Gao, 1993), semi-empirical (Roujean et al., 1992) and empirical
(Walthall et al., 1985) methods to estimate standard sun-target-sensor geometry. Various studies related to BRDF have been conducted, including Bidirectional Reflectance Factor (BRF) data measurement (Chopping, 2000; Kimes et al., 1985), retrieval of information from BRDF (Pinty and Verstraete, 1991; Privette et al., 1997; Lucht et al., 2000; Han et al., 2003 ), and BRDF model development (Roujean et al., 1992; Wanner and Strahler, 1995; Hu et al., 1997; Lacaze and Roujean, 2001). In order to correct the surface anisotropy effects, this paper used the semiempirical kernel-driven BRDF model based on of Roujean et al. (1992). The wide swath satellites like SPOT/VGT can observed reflectance with sufficient angular sampling, but high resolution satellites such KOMPSAT-2, SPOT-4, 5 and RapidEye are very difficult to obtain sufficient angular sampling over a pixel during short period because of their long term revisit period, narrow swath scanning and VZA (Viewing Zenith Angle) range within the range $0 \sim 5^{\circ}$. Therefore, BRDF parameters are difficult to directly calculate from high resolution satellites.

The purpose of this study is to be an efficiency assessment for reflectance normalization of RapidEye employing BRDF components of wide-swath satellite.

\section{Data and study area}

The VEGETATION (VGT) sensor on board the SPOT-4 platform was launched in March 1998 (http://spot-vegetation.com). The instrument observes a region of the earth $2250 \mathrm{~km}$ wide with daily coverage. The satellite has an equatorial local crossing time of 10:30 in the morning local solar time. The across track resolution is approximately 1.1 $\mathrm{km}$ at the nadir. The data are projected and interpolated to a constant pixel resolution of 
An Efficiency Assessment for Reflectance Nomalization of RapidEye Employing BRD Components of Wide-Swath Satellite

Table 1. Characteristics of SPOT/VGT and RapidEye.

\begin{tabular}{c|c|c}
\hline \multirow{2}{*}{ Mission characteristic } & \multicolumn{2}{|c}{ Information } \\
\cline { 2 - 3 } & SPOT/VGT & RapidEye \\
\hline Orbit Altitude & $820 \mathrm{~km}$ in Sun-synchronous orbit & $630 \mathrm{~km}$ in Sun-synchronous orbit \\
\hline Spectral Bands & Capable of capturing any of the following spectral bands: \\
\hline Name & $\frac{\text { Spectral Bands (nm) }}{430-470}$ & $\frac{\text { Spectral Bands (nm) }}{440-510}$ \\
\hline Blue & & $520-590$ \\
\hline Green & $610-680$ & $630-685$ \\
\hline Red & & $690-730$ \\
\hline Red Edge & $780-890$ & $760-850$ \\
\hline NIR & $1580-1750$ & \\
\hline SWIR & $1 \mathrm{~km}$ & $5 \mathrm{~m}$ \\
\hline Pixel size & & \\
\hline
\end{tabular}

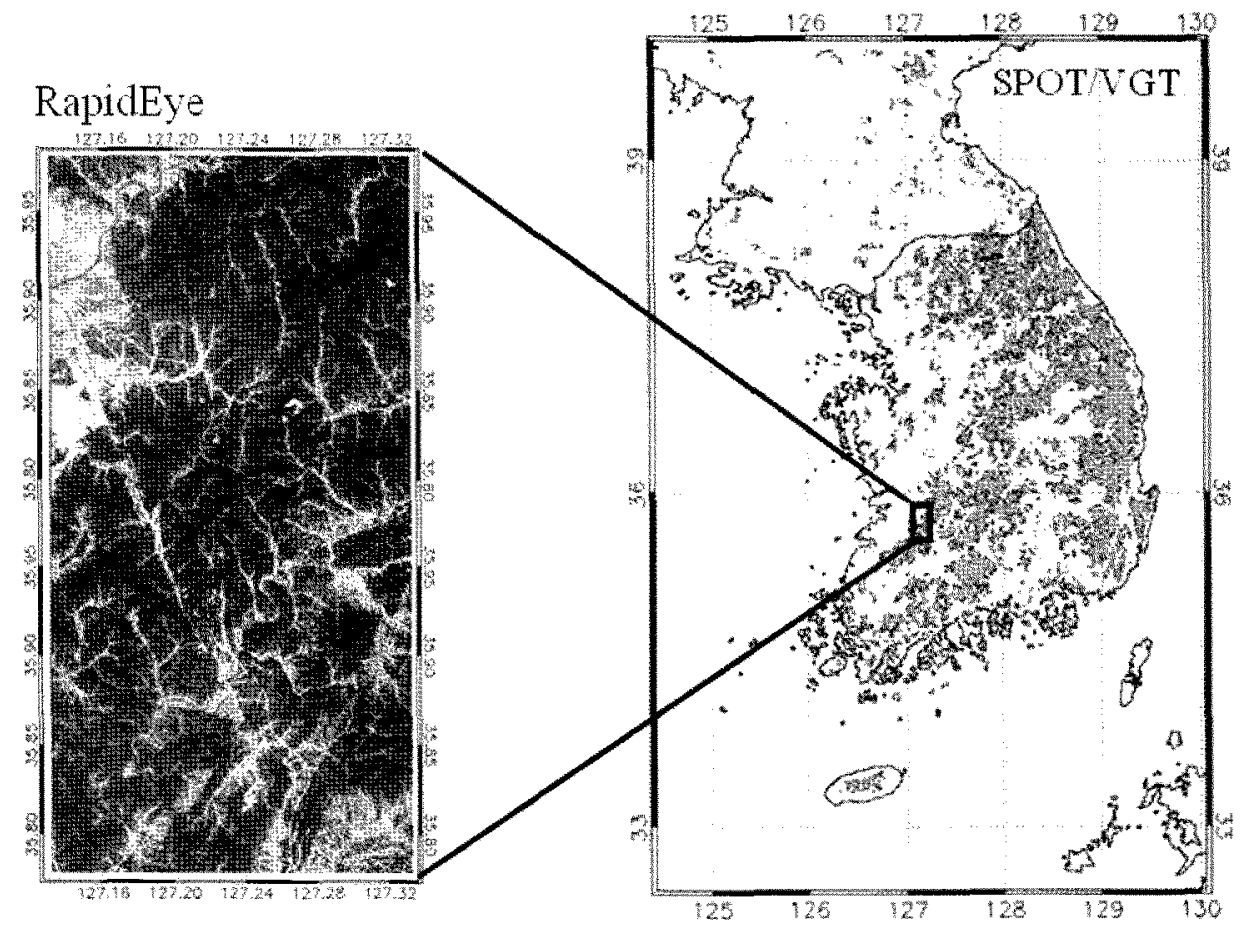

Fig. 1. The location of study area; left side: Rapideye, right side: SPOTNGT

approximately one $\mathrm{km}^{2}$. Four spectral bands are available: B0 between 0.43 and $0.47 \mu \mathrm{m}$ (blue), B2 between 0.61 and $0.68 \mu \mathrm{m}$ (red), B3 between 0.78 and $0.89 \mu \mathrm{m}$ (near infrared) and SWIR between 1.58 $-1.75 \mu \mathrm{m}$ (short wave infrared). Daily, surface reflectance (S1) products were used for this study. Also, it is optimized for vegetation observation to one of the representative polar-orbiting satellites. Besides the band, S1 product includes angle data of satellite/ sun, composite time, NDVI (Normalized Difference Vegetation Index) and SM (Status Map) data. SM data provides clear, shadow, uncertain and cloud condition to each pixel. The study was only performed when was clear. 
The high resolution satellite used within this study is the RapidEye with multispectral sensor. The RapidEye system consists of five satellites that carry the identical sensor. The RapidEye sensors have five bands in the spectral range between 0.4 and $0.9 \mu \mathrm{m}$. The features differentiating the RapidEye system from other multi-spectral sensors are for one the additional band in the red-edge region of the spectrum that allows a better analysis of vegetation, but also the high spatial resolution $(5 \mathrm{~m})$ and the high repetition rate of 1 to 3 days of the sensors due to a constellation of five RapidEye satellites. Also, atmospheric calibration was performed to use MODTRAN 4.0 by applying standard atmospheric mid-latitude profile. Table.1 shows a summary of the characteristics of the two satellites. For the analysis presented here, image of each sensor was used. For SPOT/VGT, the images were acquired on 1 January to 31 December 2009 , for RapidEye, the image was acquired on September 30th 2010. The study site is a section of Korea between $32.5^{\circ}$ and $40.0^{\circ} \mathrm{N}$ and $124.5^{\circ}$ and $130.0^{\circ} \mathrm{E}$ in SPOT/VGT. The study area of RapidEye is shown in Fig. 1. NDVI is the most widely used vegetation index in order to uncover biological characteristics of vegetation canopy (Jiang et al., 2006). NDVI is a useful index to monitoring growth of the vegetation cover. NDVI can calculate using the reflectance difference of red channel (B2) and NIR(near infra-red) channel (B3) which is maximum surface vegetation chlorophyll. Therefore, this study was performed by B2 and B3 band which are useful for monitoring in growth of vegetation cover.

\section{Methodology}

\section{1) BRDF modeling}

The schematic diagram is shown in Fig. 2. Procedure for correction the anisotropy effects of surface reflectance is required to BRDF for normalization. In addition, it is essential to estimate normalized reflectance through BRDF model to eliminate anisotropy effects. In particular, kerneldriven based semi-empirical models considered as solution about complicate relationship between surface and atmosphere radiation scattering characteristics to be in estimating BRDF. We used BRDF model developed by Roujean et al. (1992), considered to be the basic form for semi-empirical BRDF models, to simulate the BRDF parameters:

$$
\begin{gathered}
\rho\left(\theta_{s}, \theta_{v}, \phi\right)=K_{0}+K_{1} f_{1}\left(\theta_{s}, \theta_{v}, \phi\right)+K_{0}+K_{2} f_{2}\left(\theta_{s}, \theta_{v}, \phi\right) \\
f_{1}\left(\theta_{s}, \theta_{v}, \phi\right)=\frac{1}{2 \pi}[(\pi-\phi) \cos \phi+\sin \phi] \\
\times \tan \theta_{s} \tan \theta_{v}-\frac{1}{\pi}\left[\tan \theta_{s}+\tan \theta_{v}+\right. \\
\sqrt{\left.\tan ^{2} \theta_{s}+\tan ^{2} \theta_{v}+2 \tan \theta_{s} \tan \theta_{v} \cos \phi\right]} \\
f_{2}\left(\theta_{s}, \theta_{v}, \phi\right)=\frac{4}{3 \pi} \frac{1}{\cos \theta_{s}+\cos \theta_{v}} \\
\times\left[\left(\frac{\pi}{2}-\xi\right) \cos \xi+\sin \xi-\frac{1}{3}\right] \\
\cos \xi=\cos \theta_{s} \cos \theta_{v}+\sin \theta_{s} \sin \theta_{v} \cos \phi
\end{gathered}
$$

where $\rho$ denotes the reflectance in a given spectral channel. The $f_{1}$ and $f_{2}$ are two angular kernels that depend on the solar zenith angle $\theta_{\mathrm{s}}$, the viewing satellite zenith angle $\theta_{\mathrm{v}}$, and the relative azimuth angle $\phi$ between the sun and view directions. The term $K_{0}$ (isotropic value) stands for the Lambertian reflectance of the model isotropic component. The two other terms $K_{1}$ (geometric value) and $K_{2}$ (volume scattering value) are coefficients indicative of the relative contributions of $f_{1}$ and $f_{2}$, which represent, respectively, the geometric and the volume scattering effects. The three coefficients are retrieved for each channel on a pixel-per-pixel basis adjusting the model of Eq. (1) by using SPOT/VGT S1 data measurements in a less square sense. We consider sets of observations comprised within a 30 -day period to 


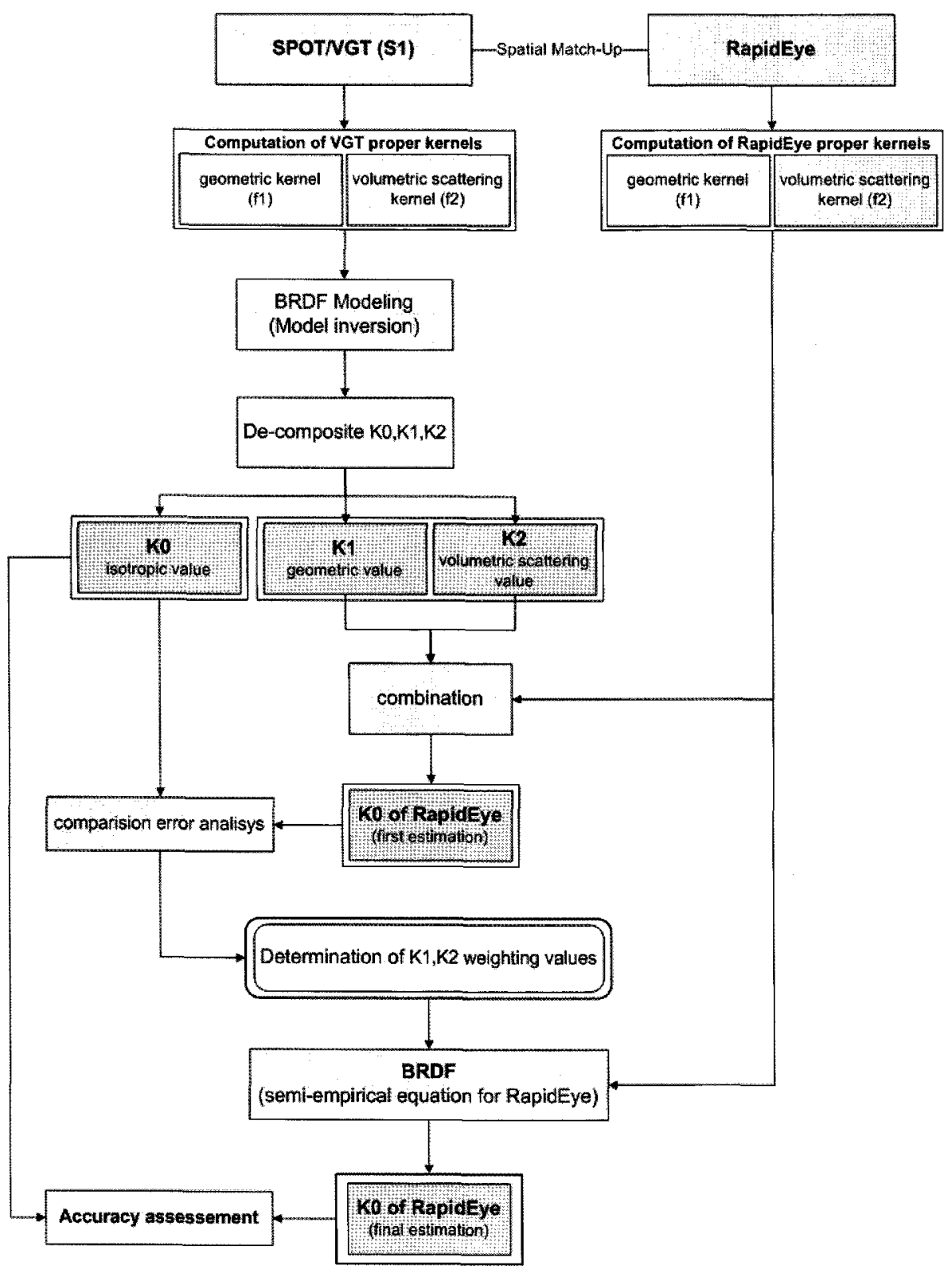

Fig. 2. Flowchart for the overall data processing of the study.

retrieve the model coefficients. The simulated reflectances are assigned to the date centred on the 30 -day period. The process is repeated with sliding periods of 10 days delineating the composite frame in order to enhance the temporal sampling (Fig. 3). The method was proven to be efficient with time series of AVHRR data (Csiszar et al., 2001; Leroy and Roujean,
1994), which provides a similar distribution to SPOT/VGT (Duchemin and Maisongrande, 2002).

\section{2) Match up database of SPOT/NGT and RapidEye}

To resolve the pixel difference of SPOT/VGT and Rapid Eye, we resampled pixel size of RapidEye to 


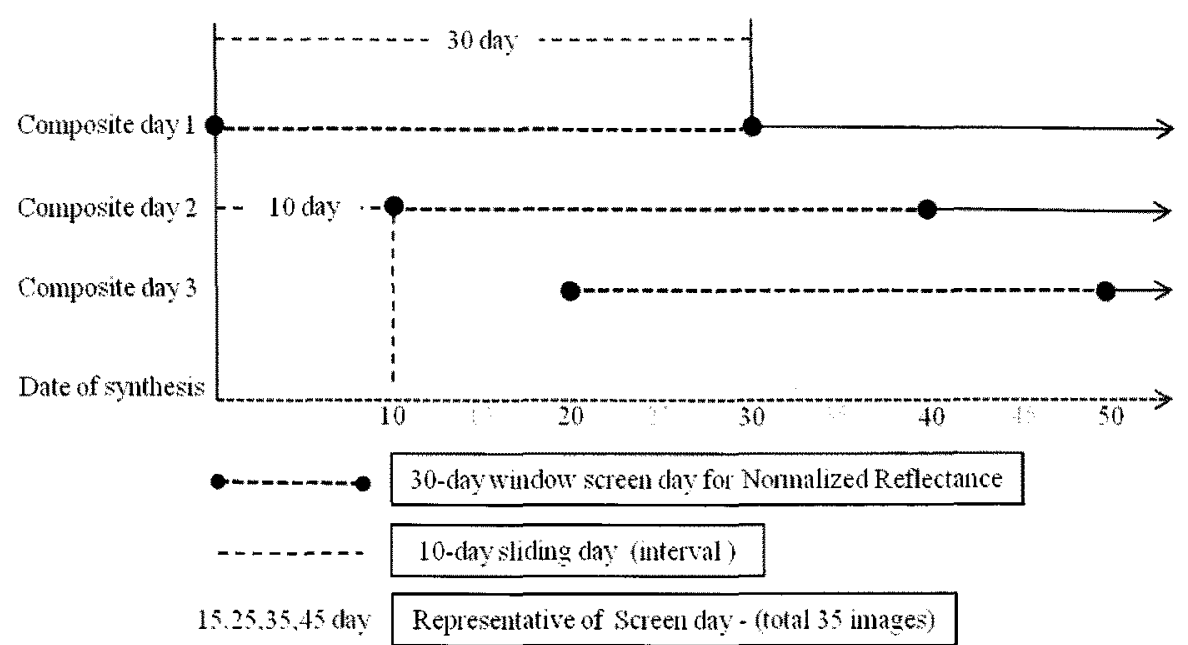

Fig. 3. The schema of method for the retrieval of normalized reflectance by using determined time window with synthetic use SPOTNGT.
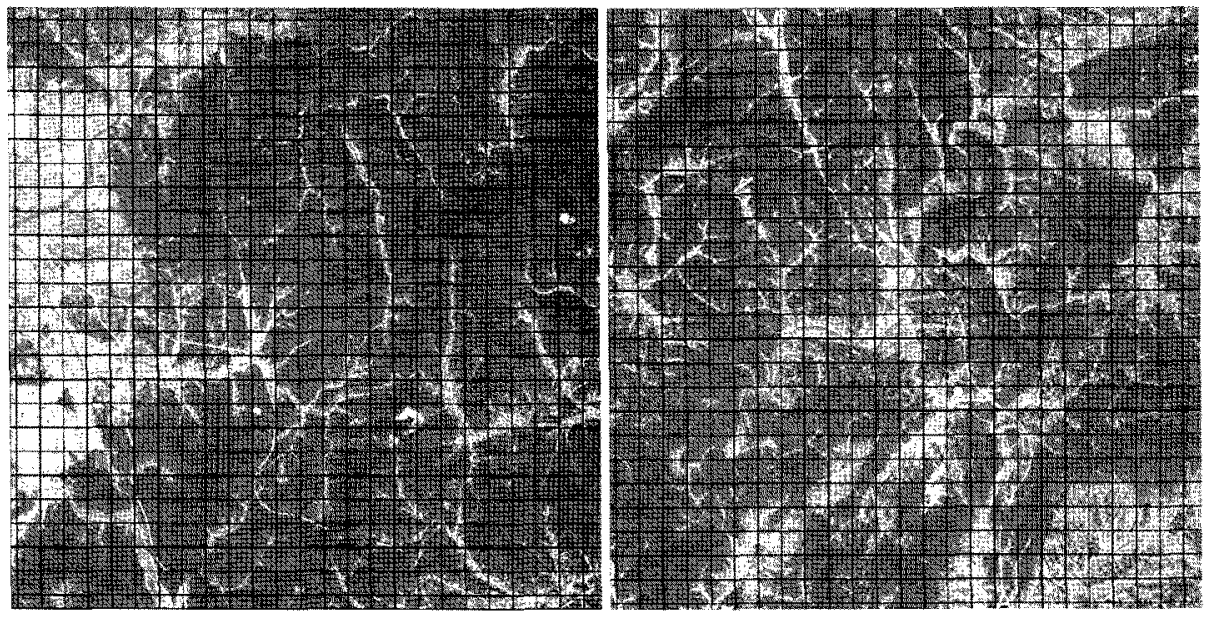

Fig. 4. A grid of RapidEye corresponding to VGT 1 pixel; (a) training scene of RapidEye; (b) test scene of RapidEye.

SPOT/VGT spatial resolution. Fig. 4 shows RapidEye image corresponding to pixel of SPOT/VGT. To solve the inconsistency between temporal and spatial patterns, RapidEye has been an average of reflectance values which is $200 \times 200$ pixels for fitting SPOT/VGT one pixel. Also, relatively large values to standard deviation were excluded. Because of relatively much more one pixel of RapidEye than one pixel of SPOT/VGT, research was intended for broaden forest area than various land surface. Add to that, forest structure is an important factor in the estimation of energy and carbon fluxes between land and atmosphere and is required by modelers regional and global scales (Zhuosen Wang et al., 2011). Also, $\mathrm{R}^{2}$ considering correlation diagram through distribution of NDVI values corresponding to RapidEye area is $0.62 . \mathrm{R}^{2}$ seems to be fair on value when compared temporal and spatial differences between the two satellites (Fig. 5). The wide swath satellites like SPOT/VGT permit sufficient angular sampling, but high resolution satellites such as IKONOS, Quickbird, and 
KOMPSAT-2 are impossible to obtain sufficient angular sampling over a pixel during short period because of their narrow swath scanning and VZA (Viewing Zenith Angle) range within the range from $0^{\circ}$ to $5^{\circ}$ degree. Consequently, in case of semiempirical BRDF model, it is difficult to parameterize kernel parameters by using high resolution satellites.

$$
\begin{gathered}
K_{0 R E}=\rho_{\mathrm{RE}}-K_{1 V G T} f_{1 R E}-K_{2 V G T} f_{2 R E} \\
K_{0 V G T}-\rho_{\mathrm{RE}}=-K_{1 V G T} f_{1 R E}-K_{2 V G T} f_{2 R E} \\
K_{0 R E}=\rho_{\mathrm{RE}}-a_{1} K_{1 V G T} f_{1 R E}-a_{2} K_{2 V G T} f_{2 R E}+a_{0}
\end{gathered}
$$

$K_{0 R E}$ is normalized reflectance at RapidEye for specific angle condition, $K_{0 V G T}$ is normalized reflectance at SPOT/VGT for specific angle condition.

Each term means following description:

$\rho_{\mathrm{RE}}$ - denotes the reflectance of RapidEye in a given spectral channel

$f_{1 R E}, f_{2 R E}$ - two angular kernels of BRDF model (in RapidEye) (depend on the solar zenith angle $\theta_{\mathrm{s}}$, the viewing satellite zenith angle $\theta_{\mathrm{v}}$, and the relative azimuth angle $\phi$ between the sun and view directions) $K_{1 V G T}, K_{2 V G T}$ - coefficients indicative of the relative contributions of kernel (geometric and volumetric scattering effects of SPOT/VGT)

Therefore, $K_{0 R E}$ is estimated by using the Eq. (5). We compared the $K_{0 V G T}$ and the estimated $K_{0 R E}$ by Eq. (5). SPOT/VGT kernels segregated proper kernel to be division and RapidEye kemels computed. We set $K_{0 V G T}-\rho_{\mathrm{RE}},-K_{1 V G T} f_{1 R E}$ and $-K_{2 V G T} f_{2 R E}$ terms corresponding to each channel (Red, NIR band) through Eq. (6); $K_{0 V G T}-\rho_{\mathrm{RE}}$ term as a dependent variable and $-K_{1 V G T} f_{1 R E}$ term, $-K_{2 V G T} f_{2 R E}$ term as the independent variables. Linear regression analysis was performed by using the parameters of SPOT/VGT like as isotropic value $\left(K_{0}\right)$, geometric value $\left(K_{1}\right)$ and volumetric scattering value $\left(K_{2}\right)$, and the kernel values of RapidEye which is geometric characteristic from observation angles, by using geometric kemel $\left(\mathrm{f}_{1}\right)$ and volumetric scattering kernel $\left(\mathbf{f}_{2}\right)$. Also it made a decision of weighting for $K_{1}, K_{2}$ and error through regression models. Expression applied weight in each of the channels is shown in Eq. (7).

\section{Comparison results}

\section{1) Preliminary comparison of SPOT/VGT and RapidEye}

Two RapidEye images with clear sky condition were collected over forest of SPOT/VGT (path24/ row24, September 30, 2010). We used Eq. (2) and Eq. (3) to calculate each of kernels which specified to RapidEye observation geometry. Input data for applying BRDF model appear Table 2. We used Eq. (5) which is modified in Eq. (1), because BRDF parameters like as $K_{0}, K_{1}$ and $K_{2}$ are difficult to directly calculate from high resolution satellites. Fig.

Table 2. Geometric and volumetric scattering Kernels for training and test scene of RapidEye.

\begin{tabular}{c|c|c}
\hline RapidEye & $\begin{array}{c}\text { Training scene } \\
(2010.09 .30)\end{array}$ & $\begin{array}{c}\text { Test scene } \\
(2010.09 .30)\end{array}$ \\
\hline SZA (solar zenith angle) & $38.4367^{\circ}$ & $38.22049^{\circ}$ \\
\hline VZA (viewing zenith angle) & $0.1747^{\circ}$ & $0.1747^{\circ}$ \\
\hline SAA (solar azimuth angle) & $178.10^{\circ}$ & $178.1215^{\circ}$ \\
\hline VAA (viewing azimuth angle) & $279.77^{\circ}$ & $279.75^{\circ}$ \\
\hline f1 (geometric kermel) & $\mathbf{- 0 . 5 0 6 1 4}$ & $\mathbf{- 0 . 5 0 2 2 4}$ \\
\hline $\mathbf{f 2}$ (volumetric scattering kernel) & $\mathbf{0 . 0 1 7 7 0}$ & $\mathbf{- 0 . 0 1 7 6 1}$ \\
\hline
\end{tabular}




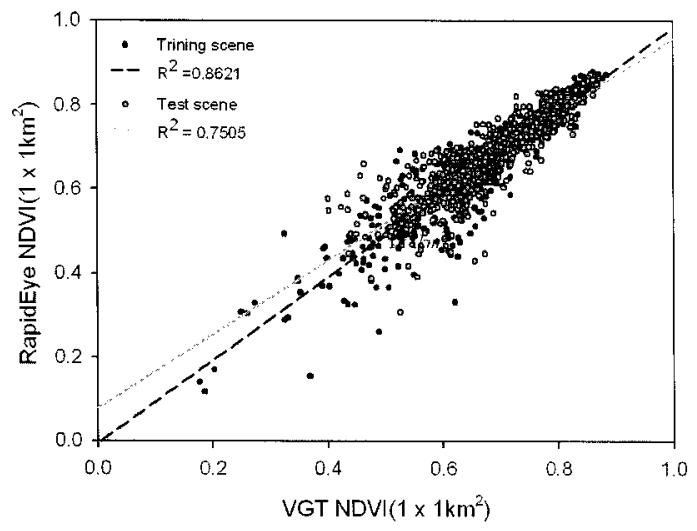

Fig. 5. NDVI comparison of SPOTNGT and RapidEye in the study area
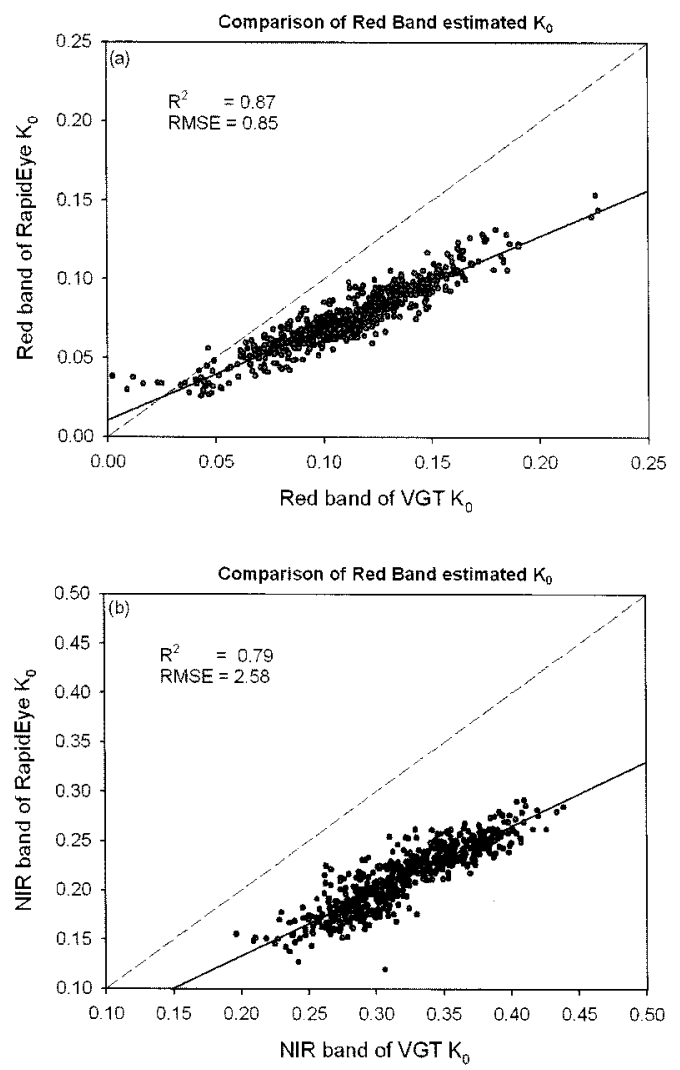

Fig. 6. Preliminary comparison of SPOT/VGT $K_{0}$ and RapidEye $K_{0}$ in the training scene; (a) Red band; (b) NIR band

6 shown preliminary comparison which is the SPOT/VGT $K_{0}$ and RapidEye $K_{0}$ in the training scene. As previously mentioned, $K_{0}$ as the normalized reflectance is closely related to the BRDF parameters (isotropic value; $K_{0}$, geometric value; $K_{1}$, volumetric scattering value; $K_{2}$ ) and the kernels (geometric kernel; $f_{1}$, volumetric scattering kernel; $f_{2}$ ). Fig. 6 shows scatter plot of the SPOT/VGT and RapidEye relationship, and shows the linear relationship between the two. The $\mathrm{R}^{2}$ value was 0.87 in Red band and 0.79 in NIR band.

$$
K_{0 V G T}=K_{0 R E}+\varepsilon
$$

RapidEye $K_{0}$ generally tended under-estimate, but we confirmed the linear relationship of the SPOT/VGT $K_{0}$ and RapidEye $K_{0}$. Eq. (8) shows the SPOT/VGT and RapidEye $K_{0}$ simplified relationship. This means that the RapidEye $K_{0}$ can be estimated from BRDF parameters of SPOT/VGT. Therefore, we performed error term analysis for directly estimating RapidEye $K_{0}$.

\section{2) Comparison BRDF $\kappa_{0}$ - adjusted weighting}

Eq. (6) shows the $K_{0 V G T}-\rho_{\mathrm{RE}},-K_{2 V G T} f_{2 R E}$ and terms corresponding to each channels $\left(K_{0 V G T}-\rho_{\mathrm{RE}}\right.$ term; a dependent variable, $-K_{1 V G T} f_{1 R E}$ and $-K_{2 V G T}$ $f_{2 R E}$ term; the independent variables). Linear regression analysis was performed by using the parameters of SPOT/VGT like as isotropic value $\left(K_{0}\right)$, geometric value $\left(K_{1}\right)$ and volumetric scattering value $\left(K_{2}\right)$, and the kernel values of RapidEye like as geometric kernel $\left(f_{1}\right)$ and volumetric scattering kernel $\left(\mathrm{f}_{2}\right)$. Also it made a decision of weighting for $K_{1}, K_{2}$ and error through regression models.

$$
\begin{aligned}
& K_{0 R E}=\rho_{\mathrm{RE}}+1.506 K_{1 V G T} f_{1 R E}-3.646 K_{2 V G T} f_{2 R E}+0.018 \\
& K_{0 R E}=\rho_{\mathrm{RE}}+1.249 K_{1 V G T} f_{1 R E}-0.983 K_{2 V G T} f_{2 R E}+0.106(10)
\end{aligned}
$$

We estimated RapidEye $K_{0}$ about Red band using Eq. (9) and NIR band using the Eq. (10). The results of a correlation analysis between the SPOT/VGT $K_{0}$ and the applying weighted RapidEye $K_{0}$, shown in Fig. 7. Fig. 7 shows that correlation existed (RMSE= 0.001929 , bias $=0.00008032$ in Red band, and 

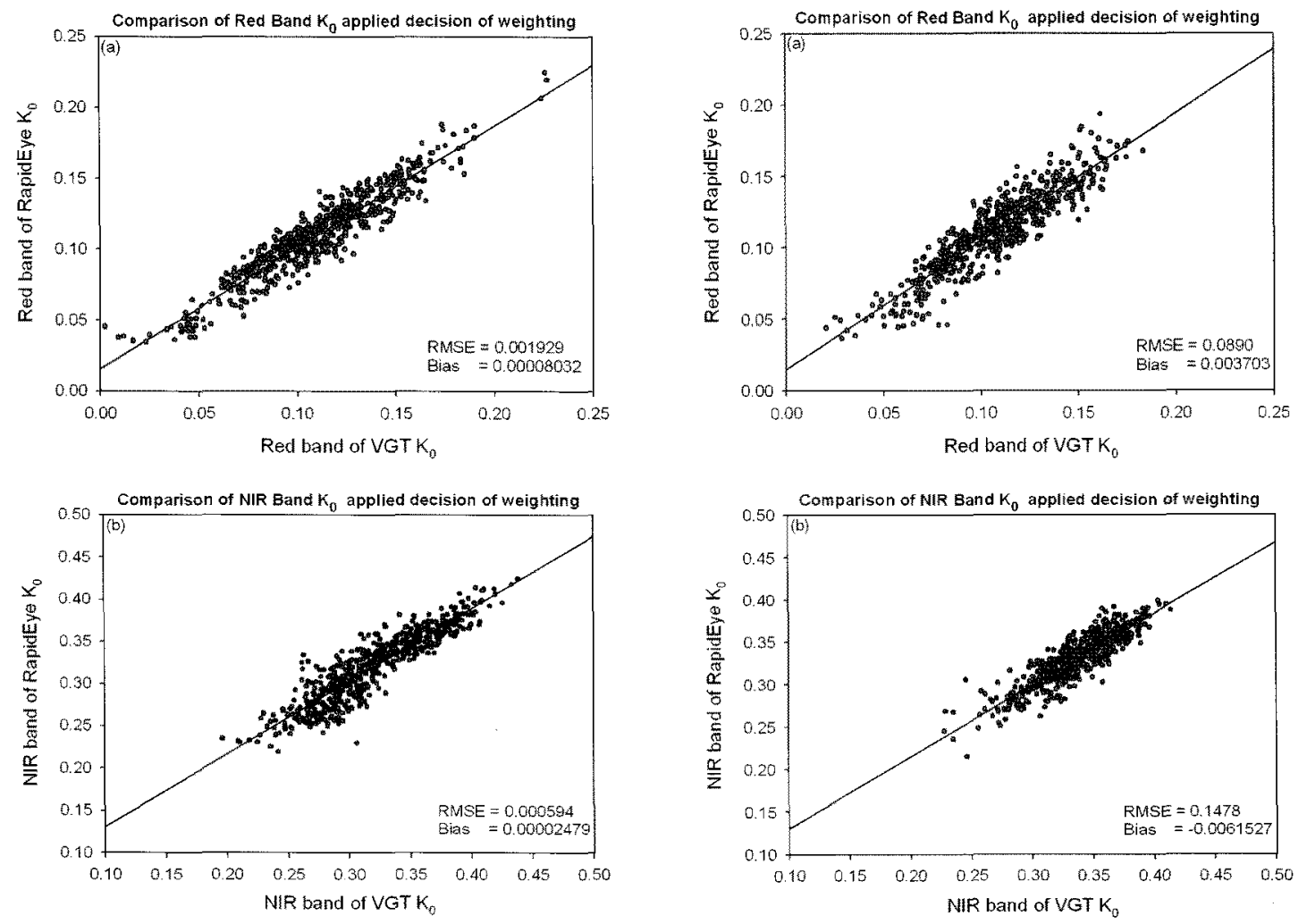

Fig. 7. Comparison of SPOT/VGT $K_{0}$ and RapidEye $K_{0}-$ adjusted weighting $K_{0}$ in the training scene; (a) Red band; (b) NIR band

Fig. 8. Comparison of SPOT/VGT $K_{0}$ and RapidEye $K O$ adjusted weighting $K_{0}$ in the test scene; (a) Red band; (b) NIR band

Table 3. Result (Bias and RMSE) of correlation analysis between SPOTNGT $K_{0}$ and RapidEye $K_{0}$ about each of the channels

\begin{tabular}{|c|c|c|c|c|c|c|}
\hline \multirow[b]{2}{*}{ Red band } & \multicolumn{2}{|c|}{$\begin{array}{c}\text { RapidEye } K_{0} \\
\text { (Training scene) }\end{array}$} & \multicolumn{2}{|c|}{$\begin{array}{l}\text { RapidEye } K_{0} \\
\text { (Test scene) }\end{array}$} & \multicolumn{2}{|c|}{ Total } \\
\hline & NIR band & Red band & NIR band & Red band & NIR band & \\
\hline Bias & 0.000080 & 0.000025 & 0.003703 & -0.0061527 & 0.0018915 & -0.0030638 \\
\hline RMSE & 0.001929 & 0.005940 & 0.0890 & 0.1478 & 0.0454645 & 0.07687 \\
\hline
\end{tabular}

RMSE $=0.000594$, bias $=0.00002479$ in NIR band). To assess the accuracy of the weighting, we performed Eq. (9) and Eq. (10) over the test scene. Fig. 8 shows the result which is $\mathrm{RMSE}=0.0890$, bias $=0.003703$ in Red band, and RMSE $=0.1478$, bias $=-0.0061527$ in NIR band. As a result, the weighting through linear regression analysis produced good agreement. For all sites, the SPOT/VGT $K_{0}$ and RapidEye $K_{0}$ had the high correlation (RMSE, bias), and generally were very consistent. We also compared with two area estimated to be using the weighting. Table 3 provided the detailed information about the estimated RapidEye $K_{0}$ accuracies. The final estimated RapidEye $K_{0}$ was batter than that in preliminary comparison result, as supported by statistical results. Through weighted RapidEye $K_{0}$ by error term analysis, we estimated the RapidEye $K_{0}$ directly using the SPOTNGT. However, errors in the estimated the RapidEye $K_{0}$ directly using the SPOT/VGT were 

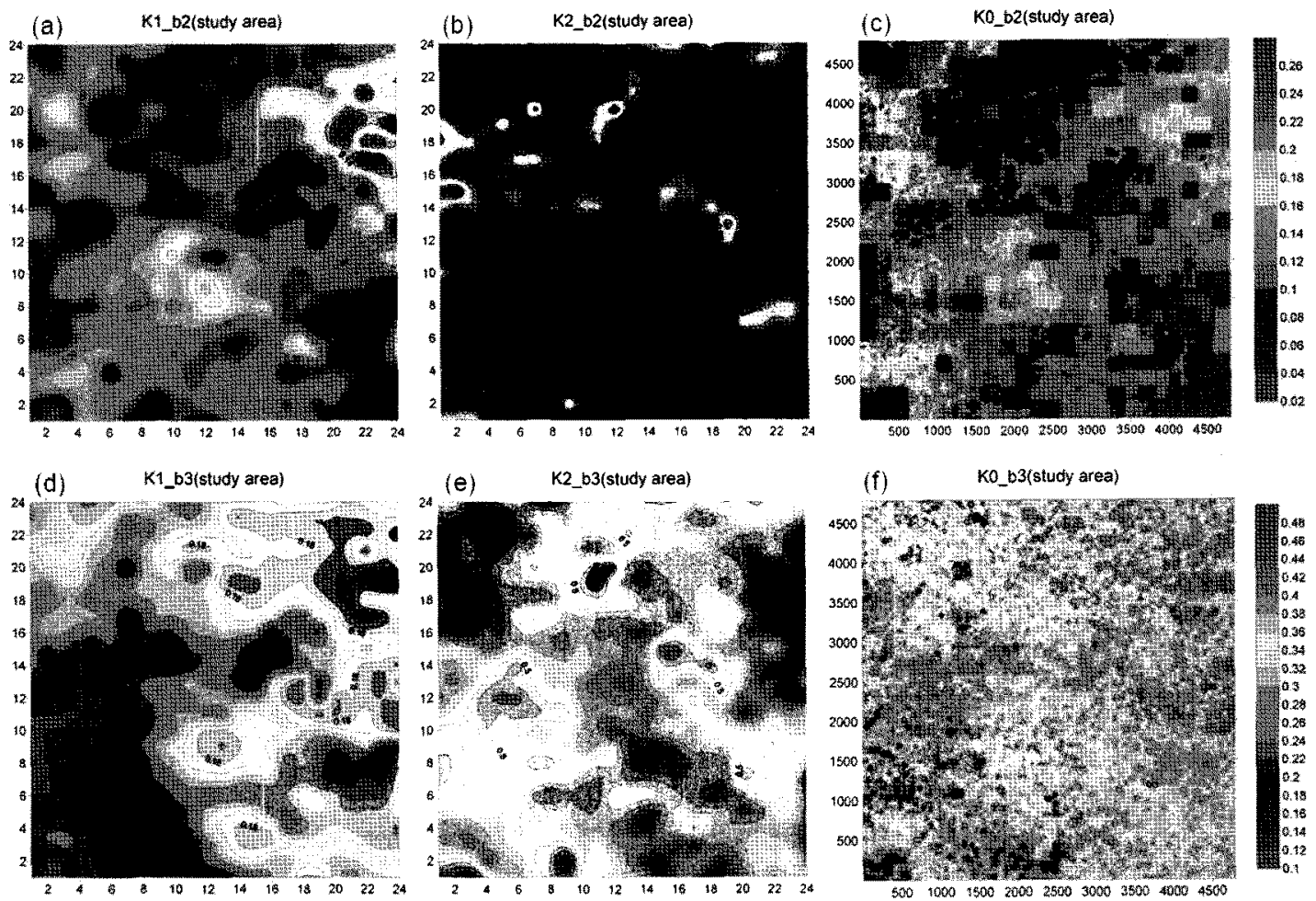

Fig. 9. $K_{1}$ image applied weighting, $K_{2}$ image applied weighting and estimated $K_{0}$ image over the training scene: (a) Red band of geometric value; (b) Red band of volumetric scattering value; (c) Red band of estimated isotropic value; (d) NIR band of geometric value; (e) NIR band of volumetric scattering value; (f) NIR band of estimated isotropic value.

observed without improved processing, and it was focused on some parts of the area. We also compared our results to those of other method (Fig. 9). Fig. 9 shown $K_{1}$ image applied weighting, $K_{2}$ image applied weighting and estimated $K_{0}$ image over the training scene. Two values of $K_{1}$ and $K_{2}$ are in inverse proportion to each other. $K_{1}$ is in direct proportion to the estimated $K_{0}$, and $K_{2}$ is in inverse proportion to the $K_{0} . K_{0}$ seem to affects more $K_{2}$ values than $K_{1}$ values in the forest. In the future, we will apply weighting to various land cover about RapidEye reflectance.

\section{Concluding remarks}

We tested the performance of the SPOT/VGT BRDF components when estimated RapidEye $K_{0}$. Typically, the wide swath satellites like SPOT/VGT permit sufficient angular sampling, but high resolution satellites are impossible to obtain sufficient angular sampling over a pixel during short period because of their narrow swath scanning and VZA (Viewing Zenith Angle) range within the range $0 \sim 5^{\circ}$. Consequently, BRDF parameters are difficult to directly calculate from high resolution satellites. To solve this problem, we performed linear regression analysis by using the parameters of SPOT/VGT like as isotropic value $\left(K_{0}\right)$, geometric value $\left(K_{1}\right)$ and volumetric scattering value $\left(K_{2}\right)$, and the kernel 
values of RapidEye like as geometric kernel $\left(f_{1}\right)$ and volumetric scattering kernel $\left(\mathrm{f}_{2}\right)$. Also it made a decision of weighting for $K_{1}, K_{2}$ and error through regression models. We compared the SPOT/VGT $K_{0}$ estimates with comparison RapidEye $K_{0}$ to assess their accuracy. Correlation existed (RMSE= 0.001929 , Bias $=0.00008032$ in Red band, and RMSE $=0.000594$, Bias $=0.00002479$ in NIR band). To assess the accuracy of the weighting, test scene shows the result which is $\mathrm{RMSE}=0.089$, Bias= 0.003703 in Red band, and RMSE $=0.1478$, Bias $=$ -0.0061527 in NIR band. As a result, the weighting through linear regression analysis produced good agreement. For all sites, the SPOT/VGT $K_{0}$ and RapidEye $K_{0}$ had the high correlation (RMSE, Bias), and generally were very consistent. As a result, the weighting through linear regression analysis produced good agreement. For all sites, the SPOT/VGT and RapidEye had the high correlation (RMSE, Bias), and generally were very consistent. We also compared with two scene estimated to be using the weighting. However, this study has several limitations in simple comparison about reflectance of RapidEye and SPOT/VGT, and in efficiency assessment test. In the future, this researcher will be utilized as basic data for estimating normalized reflectance of high resolution satellite and we will apply weighting to various land cover about RapidEye reflectance.

\section{Acknowledgements}

This subject is supported by Ministry of Environment as "The Eco-technopia 21 project" (121-091-069).

We thank the Korea Aerospace Research Institute (KARI) for providing the RapidEye data used for sole research purpose.

\section{References}

Chopping, M. J., 2000. Testing a LiSK BRDF model with in situ bidirectional reflectance factor measurements over semiarid grasslands. Remote Sensing of Environment, 74: 287-312.

Csiszar, I., G. Gutman, P. Romanov, M. Leroy, and O. Hautecoeur, 2001. Using ADEOS/ POLDER data to reduce angular variability of NOAA/AVHRR reflectances. Remote Sensing of Environment, 76: 399-409.

Dickinson, R. E. 1983. Land surface processes and climate surface albedos and energy balance. Advances in Geophysics, 25, 305-353.

Duchemin, B. and P. Maisongrande, 2002. Normalization of directional effects in 10-day global syntheses derived from VEGETATION/ SPOT: I. Investigation of concepts based on simulation. Remote Sensing of Environment, 81: 90-100.

Gao, W., 1993. A simple bidirectional-reflectance model applied to a tallgrass canopy. Remote Sensing of Environment, 45: 209-224.

Han, K. S., J. L. Champeaux, J. L. Roujean, 2004, A land cover classification product over France at $1 \mathrm{~km}$ resolution using SPOT4/VEGETATION data. Remote Sensing of Environment, 92(1): 52-66.

Hu, B., W. Wanner, X. Li, and A. H. Strahler, 1997. Validation of kernel-driven semi-empirical models for global modeling of bidirectional reflectance. Remote Sensing of Environment, 62: 201-214.

Jiang, Z., A. R. Huete, J. Chen, Y. Chen, Li, G. Yan and X. Zhang, 2006. Analysis of NDVI and scaled difference vegetation index retrievals of vegetation fraction. Remote Sensing of Environment, 101(3): 366-378.

Yeom, J. M., K. S. Han, and Y. S. Kim, 2005. A Reflectance Normalization Via BRDF Model 
for the Korean Vegetation using MODIS $250 \mathrm{~m}$ Data. Korean Journal of Remote Sensing, 21(6): 361-368

Yeom, J. M., K. S. Han, 2009. An Efficiency Analysis for Data Synthesis of Sun- and GeoSynchronous Satllites in kernel-driven BRDF Model. Asia-Pacific Journal of Atmospheric, 45(4): 499-511

Kimes, D. S., W. W. Newcombe, C. J. Tucker, I. W. Zonnefeld, W. van Vijingaarden, J. de Leeuw, and G. F. Epema, 1985. Directional reflectance factor distributions for cover types of Northern Africa. Remote Sensing of Environment, 18: 1-19.

Lacaze, R., and J. L. Roujean, 2001. G-function and HOT SPOT (GHOST) reflectance model application to multi-scale airborne POLDER measurements. Remote Sensing of Environment, 76: 67-80.

Lucht, W., C. B. Schaaf, and A. H. Strahler, 2000. An algorithm for the retrieval of albedo from space using semiempirical BRDF models. IEEE Transactions on Geoscience and Remote Sensing, 38: 977-998.

Pinty, B., and M. M. Verstraete, 1991. Extracting information on surface properties from bidirectional reflectance measurements. Journal of Geophysical Research, 96: 28652879.

Pokrovsky, O., and J. L. Roujean, 2002. Land surface albedo retrieval via kernel-based $\mathrm{BRDF}$ modeling: II. An optimal design scheme for the angular sampling. Remote Sensing of Environment, 84: 120-142.

Privette, J. L., T. F. Eck, and D. W. Deering, 1997. Estimating spectral albedo and nadir reflectance through inversion of simple BRDF models with AVHRR/ MODIS-like data. Journal of Geophysical Research, 102: 2952929542.

Roujean, J. L, M. Leroy, and P. Y. Deschamps, 1992. A bidirectional reflectance model of the earth's surface for the correction of remote sensing data. Journal of Geophysical Research, 97 (D18): 20455-20468.

Tarpley, J. D., S. R. Schneider, and R. L. Money, 1984. Global vegetation indices from the NOAA-7 meteorological satellite. Journal of Applied Meteorology, 23: 491-494.

Walthall, C. L., 1985. A study of reflectance anisotropy and canopy structure using a simple empirical model. Remote Sensing of Environment, 61: 118-128.

Wanner, W., and A. H. Strahler, 1995. On the derivation of kernels for kernel-driven models of bidirectional reflectance. Journal of Geophysical Research, 100: 21077-21089.

Zhuosen, W., S. Crystal B., L. Philip , K. Yuri, S. Mitchell A., S. Alan H., Y. Tian, M. Ranga B., C. Mark J., B. Bryan J., 2011. Retrieval of canopy height using moderate-resolution imaging spectroradiometer (MODIS) data. Remote Sensing of Environment, 115: 15951601. 